\title{
Comparison of perioperative and oncological outcomes between video-assisted segmentectomy and lobectomy for patients with clinical stage IA non-small cell lung cancer: a propensity score matching study
}

\author{
Cheng-Yang Song, Takehiro Sakai, Daisuke Kimura, Takao Tsushima, Ikuo Fukuda \\ Department of Thoracic and Cardiovascular Surgery, Hirosaki University Graduate School of Medicine, Hirosaki, Japan \\ Contributions: (I) Conception and design: I Fukuda, T Sakai; (II) Administrative support: T Sakai; (III) Provision of study materials or patients: T \\ Sakai, D Kimura, T Tsushima, I Fukuda; (IV) Collection and assembly of data: CY Song, T Sakai, D Kimura; (V) Data analysis and interpretation: \\ CY Song, T Sakai; (VI) Manuscript writing: All authors; (VII) Final approval of manuscript: All authors. \\ Correspondence to: Takehiro Sakai, MD, PhD. Department of Thoracic and Cardiovascular Surgery, Hirosaki University Graduate School of Medicine, \\ 5 Zaifu-cho, Hirosaki, Aomori 036-8562, Japan. Email: t-sakai@hirosaki-u.ac.jp.
}

Background: Segmentectomy for lung cancer remains controversial because of the complexity of the procedure and concern about an increased recurrence rate. It is important to compare perioperative and oncological outcomes between segmentectomy and lobectomy.

Methods: From January 2007 to December 2016, 41 segmentectomies by video-assisted thoracic surgery (VATS) and 122 VATS lobectomies for 163 patients with clinical stage IA non-small cell lung cancer (NSCLC) were performed. Clinicopathological factors, including recurrence rate and survival rate, were compared. In order to reduce biases of outcomes, clinicopathological factors were used for propensity score matching (PSM). Then, 41 VATS segmentectomies and 41 lobectomies were selected and further analyzed.

Results: No significant differences were seen between the two groups in age, pulmonary function, comorbidity, operative time, blood loss, chest tube duration days, postoperative stay days, complications, histological type, and multiple primary rate. Smoking index resected number of nodes, tumor size, lymph node metastasis rate, and pathological stage were higher in the lobectomy group than in the segmentectomy group $(\mathrm{P}<0.05)$. In the lobectomy group, 16 patients $(13.1 \%)$ had recurrence, and 2 patients $(1.6 \%)$ died because of cancer progression. There were no significant differences in the recurrence rate and prognosis between the two groups. In addition, Cox regression analysis suggested that sex, lymph node metastasis, and pathology stage were associated with recurrence $(\mathrm{P}<0.05)$, but no factor was an independent prognostic factor. After PSM, the two groups had similar clinicopathological factors, and the type of operation still had no relationship with the recurrence rate or the death rate.

Conclusions: Perioperative and oncological outcomes of VATS segmentectomy are similar to those of VATS lobectomy for patients with clinical stage IA NSCLC. VATS segmentectomy can be considered one of the surgical procedures appropriate for patients with clinical stage IA NSCLC.

Keywords: Non-small cell lung cancer (NSCLC); video-assisted thoracic surgery (VATS); segmentectomy; survival analysis

Submitted Apr 29, 2018. Accepted for publication Jul 28, 2018.

doi: $10.21037 /$ jtd.2018.07.133

View this article at: http://dx.doi.org/10.21037/jtd.2018.07.133 


\section{Introduction}

In the surgical treatment of early-stage non-small cell lung cancer (NSCLC), thoracic surgeons are constantly trying to find the balance between minimal invasion and radical resection. Minimally invasive technology is mainly reflected by operative incision length and resection range. Videoassisted thoracic surgery (VATS) technology has guided thoracic surgical procedures into a new stage because of innovation related to the operative incision.

In order to achieve less invasiveness, the resection range has been discussed. In 1995, the Lung Cancer Study Group (LCSG) reported a prospective, randomized, multi-institutional trial comparing lobectomy to sublobar resection for T1N0 NSCLC. They found that the limited resection group had a three times higher local recurrence rate and higher death rate than the lobectomy group (1). Smith reported that wedge resection caused a higher recurrence rate than segmentectomy (2). We considered that there might be potential biases in the LCSG study, because segmentectomy and wedge resection were not analyzed separately. Thoracoscopic lobectomy with systematic mediastinal lymph node dissection has been a standard treatment for early-stage NSCLC for many years; however, segmentectomy remains a controversial topic because of the complexity of the procedure and the fear of an increased recurrence rate. Some published studies suggested that the patient outcomes of thoracoscopic lobectomy and segmentectomy procedures for clinical stage I NSCLC were equivalent (3-5). However, Khullar et al. suggested that both wedge resection and segmentectomy were associated with worse outcomes than lobectomy (6). Whitson et al. indicated that lobectomy confers a significant survival advantage over segmentectomy (7). In order to confirm the reliability of VATS segmentectomy, more precise clinical investigation is needed.

The objective of this study was to investigate the clinical significance of VATS segmentectomy by retrospective analysis. Perioperative factors and oncological outcomes were compared between VATS segmentectomy and lobectomy in patients with clinical IA NSCLC.

\section{Methods}

\section{Patients}

A total of 163 patients who underwent VATS lobectomy (lobectomy group) or VATS segmentectomy (segmentectomy group) in the Hirosaki University Hospital from January
2007 to December 2016 were included in the present study. Patients' data were retrospectively collected from medical records. Anatomical pulmonary resection and systematic lymph node dissection were performed for each patient in this retrospective study as the standard surgical procedure. The inclusion criteria were as follows. Patients' clinical stage was cT1N0M0, and pathology was primary NSCLC. All patients received preoperative high-resolution computed tomography (HRCT) and $87.7 \%$ patients received preoperative [F-18]-fluorodeoxyglucose (FDG)positron emission tomography-computed tomography (PET-CT) and maximum standardized uptake value of FDG (SUVmax) were evaluated. Mediastinoscopy or endobronchial ultrasonography was not routinely performed. Clinical stage was determined by HRCT and PET-CT with FDG. Patients who underwent neoadjuvant chemotherapy or radiotherapy before operation were excluded. Patients with incompletely resected tumors (R1 or R2) were also excluded. Patients were staged according to the TNM Classification of Malignant Tumors, 8th Edition (8). Pathologists who were experts in lung cancer made the pathological diagnoses. The rate of multiple primary lung cancer (MPLC), which means that more than one primary lung cancer was found in resected lung tissue by the pathologists, was analyzed. VATS segmentectomy was allowed in cases of complete removal of the disease, using the optional procedure instead of lobectomy for a peripheral tumor. Both segmentectomy and multiple segmentectomies were included in the segmentectomy group. Cases of conversion from segmentectomy to lobectomy during operation were included in the lobectomy group. All patients who underwent segmentectomy were suitable for lobectomy, and all patients who underwent lobectomy were technically suitable for segmentectomy. Cases of conversion from VATS to open surgery during operation were included in the present study. Patients who were pathologically diagnosed with lymph node metastasis received platinum-based chemotherapy postoperatively.

This study was approved by The Committee of Medical Ethics of Hirosaki University Graduate School of Medicine, Hirosaki, Japan (approval number 2017-1155).

\section{Operative technique}

The patients were placed in the lateral decubitus position for thoracotomy. All surgical procedures were performed under general anesthesia with double-lumen endotracheal tube intubation. All procedures were performed by 
VATS. The first port was placed in the fourth or fifth intercostal space as the main operating port. The second port, the camera port, was usually located at the seventh or eighth intercostal space, through which a 30 -degree thoracoscope was positioned. If necessary, the third port was set in the sixth intercostal space in the posterior axillary line. Systematic lymph node dissection was performed for all patients. The parenchymal surgical margin in segmentectomy was at least $2 \mathrm{~cm}$.

\section{Follow-up}

Each patient was followed up by CT every year for at least 5 years after surgery. The deadline for follow-up was December 2017. Whenever recurrence was suspected, the interval of follow-up would be shortened, and recurrence was determined by pathological or unequivocal radiological results. Operative mortality was defined as any death within the first 30 days after operation or during the same hospitalization. Disease-free survival (DFS) and overall survival (OS) were defined as the time from the date of operation to recurrence and death, respectively.

\section{Propensity score matching (PSM)}

The propensity scores were analyzed by logistic regression models, and the matched factors included: sex, PET-CT, tumor size, lymph node metastasis, pathological stage, and previous cancer. The variables chosen for PSM were not related to the surgery, but had potential associations with short and longterm outcomes, especially long-term outcomes. Through the matching procedure for propensity scores, the segmentectomy and lobectomy groups showed similar distributions of propensity scores, indicating that the differences in covariates between the two groups were minimized. Propensity scores were matched one by one using nearest neighbor methods, no replacement, no caliper width, and match 1:1.

\section{Statistical analyses}

Statistical analyses were carried out using the Statistical Package for the Social Sciences (SPSS) software (version 22, IBM, Armonk, NY, USA). Student's $t$-test or the Wilcoxon rank-sum test was used to compare continuous variables. The $\chi^{2}$ test or Fisher's exact test was used to compare categorical variables. OS and DFS were analyzed by the Kaplan-Meier method and the log-rank test. Univariate and multivariate Cox regression analyses were performed to identify predictors of DFS. Significance was accepted as a $\mathrm{P}$ value less than 0.05 for all analyses.

\section{Results}

A total of 163 patients were analyzed in this study. Forty-one patients underwent VATS segmentectomy, and 122 patients underwent VATS lobectomy. The clinicopathological characteristics of the segmentectomy group and the lobectomy group are shown in Table 1. There were no significant differences in age, sex, smoking index, pulmonary function, and comorbidity rate between the groups. The proportion of female in segmentectomy group was higher than lobectomy group $(\mathrm{P}=0.047)$. Thirty-eight patients in the segmentectomy group and 105 patients in the lobectomy group were performed PET-CT before operation. The population of SUVmax $>2.5$ was lower in the segmentectomy group than in the lobectomy group $(\mathrm{P}=0.001)$. The previous cancer rate was higher in the segmentectomy group than in the lobectomy group $(\mathrm{P}<0.001)$. The histological type and MPLC rate were not significantly different between the two groups. The lobectomy group had significantly larger tumor size $(\mathrm{P}<0.001)$ and higher rates of node involvement $(\mathrm{P}=0.038)$ and pathologically advanced stage $(\mathrm{P}<0.001)$ than the segmentectomy group. There were no conversions from VATS to open surgery or from segmentectomy to lobectomy. There was no 30-day postoperative mortality. The two groups had no significant differences in operative time, blood loss, chest tube duration, postoperative stay, and postoperative complications. The number of resected lymph nodes was significantly higher in the lobectomy group than in the segmentectomy group $(\mathrm{P}<0.001)$. The median follow-up duration after surgery was 41 months in the segmentectomy group and 46 months in the lobectomy group, with no significant difference. Recurrence was seen in 2 patients in the segmentectomy group (4.9\%) and 16 patients in the lobectomy group (13.1\%), with no significant difference. The two patients with recurrence in the segmentectomy group were consisted of 1 pleural dissemination and lymph node recurrence and 1 pleural dissemination and pulmonary metastasis. The 16 patients with recurrence in lobectomy group included 4 lymph nodes (3.3\%), 10 lung $8.2 \%), 2$ bone (1.6\%) and 2 brain metastasis $(1.6 \%)$. Two patients in the lobectomy group died because of cancer progression.

The anatomical distributions of segmentectomy and lobectomy are shown in Table 2. Postoperative complications 
Table 1 Clinicopathological characteristics of the patients' group

\begin{tabular}{|c|c|c|c|}
\hline Variable & Segmentectomy $(n=41)$ & Lobectomy $(n=122)$ & $P$ value \\
\hline Sex & & & 0.047 \\
\hline Male & 13 & 61 & \\
\hline Female & 28 & 61 & \\
\hline PET-CT & & & $<0.001$ \\
\hline SUVmax $\leq 2.5$ & $30[38]$ & 50 [105] & \\
\hline SUVmax $>2.5$ & $8[38]$ & 55 [105] & \\
\hline \multicolumn{4}{|c|}{ Pulmonary function (mean \pm SD) } \\
\hline FEV1 & $2.38 \pm 0.63$ & $2.51 \pm 0.65$ & 0.263 \\
\hline$\%$ of predicted & $114.7 \pm 16.9$ & $111.9 \pm 16.6$ & 0.370 \\
\hline Previous cancer & $13(31.7 \%)$ & $9(7.4 \%)$ & $<0.001$ \\
\hline Comorbidity & $19(46.3 \%)$ & $67(54.9 \%)$ & 0.370 \\
\hline COPD & $6(14.6 \%)$ & $15(12.3 \%)$ & 0.788 \\
\hline CAOD & $1(2.4 \%)$ & $4(3.3 \%)$ & 1.000 \\
\hline Hypertension & $15(36.6 \%)$ & $46(37.7 \%)$ & 1.000 \\
\hline Diabetes & $4(9.8 \%)$ & $18(14.8 \%)$ & 0.598 \\
\hline No & 41 & 110 & \\
\hline $\mathrm{N} 1+\mathrm{N} 2$ & 0 & 12 & \\
\hline Stage & & & $<0.001$ \\
\hline 0 & 1 & 0 & \\
\hline $\mid \mathrm{A} 1$ & 3 & 4 & \\
\hline IA2 & 24 & 36 & \\
\hline IA3 & 7 & 70 & \\
\hline IB & 4 & 0 & \\
\hline$\| A$ & 0 & 0 & \\
\hline IIB & 2 & 3 & \\
\hline IIIA & 0 & 9 & \\
\hline
\end{tabular}

Table 1 (continued) 
Table 1 (continued)

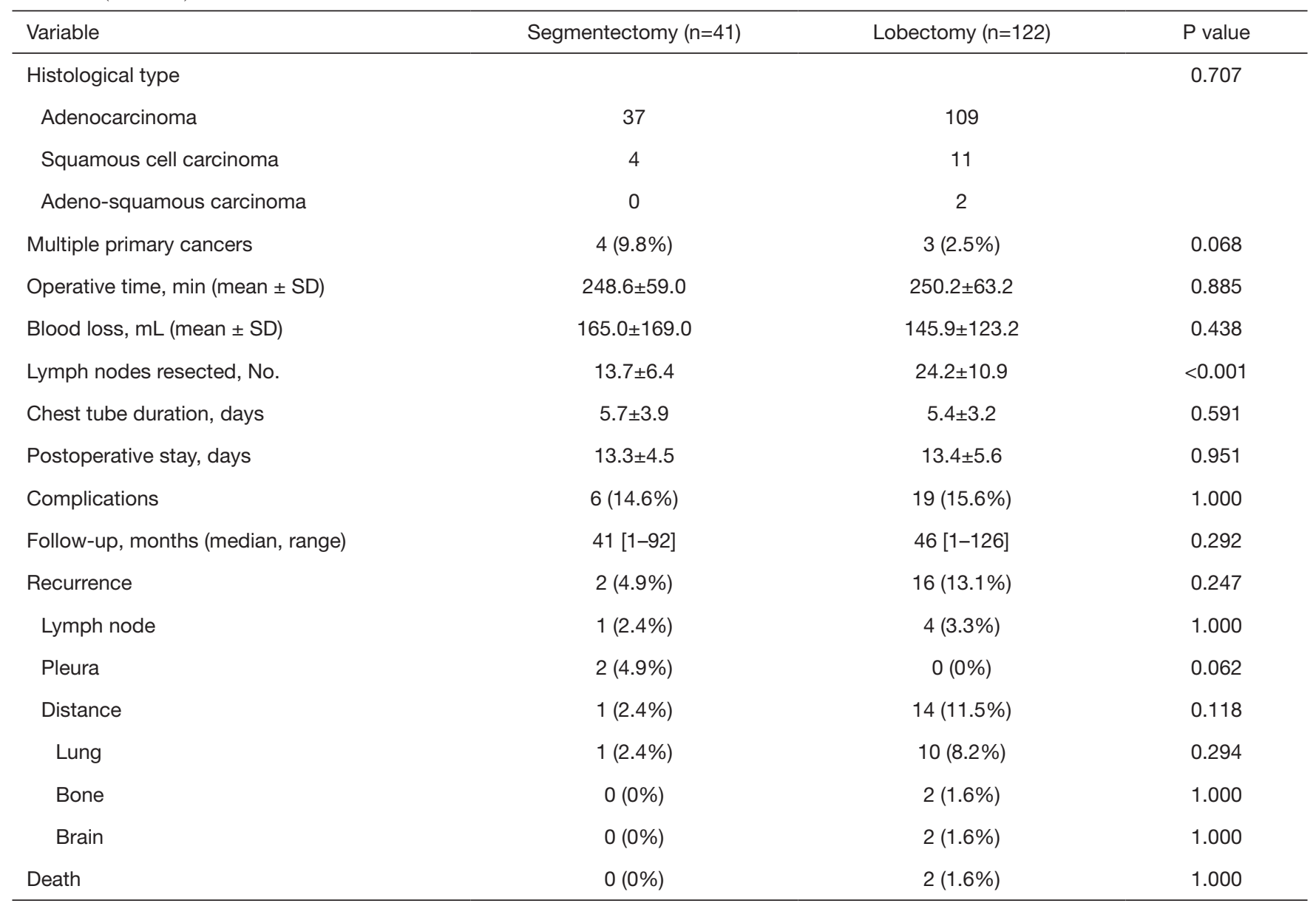

Smoking index, the average root number per day multiplied by smoking years of smoking; comorbidity: some cases are duplicated. PET-CT, positron emission and computer tomography; SUVmax, maximum standardized uptake value of [F-18]-fluorodeoxyglucose; FVC, forced vital capacity; FEV1, forced expiratory volume in one second; Dlco, diffusion capacity of the lung for carbon monoxide; COPD, chronic obstructive pulmonary disease; CAOD, coronary artery occlusive disease.

are shown in Table 3. The incidence of any postoperative complication was $14.6 \%$ in the segmentectomy group and $15.6 \%$ in the lobectomy group. No significant difference was seen between the two groups in all specific major complications.

The survival curves prepared by the Kaplan-Meier method of the two groups are shown in Figure 1. No significant difference was seen between the two groups in the DFS rate.

In order to determine whether operative procedure was related to prognosis, univariate and multivariate Cox regression analyses were performed, and their results are shown in Table 4. Univariate Cox regression analyses of DFS for all 163 patients showed that $\mathrm{N}$ stage $(\mathrm{P} \leq 0.001)$, and TNM stage $(\mathrm{P} \leq 0.001)$ were risk factors for late recurrence, whereas type of operation had no effect on recurrence.
Multivariate Cox regression analyses showed that no factor was an independent prognostic factor.

The results of PSM are shown in Table 5. Sex, PET-CT, tumor size, lymph node metastasis, pathological stage, and previous cancer were used for PSM, and 41 segmentectomies and 41 lobectomies were included in the cohort for further analysis by PSM. The results showed that the clinicopathological factors were not significantly different between the two groups, including the recurrence rate and death rate. These results mean that, with balanced characteristics, type of operation still had no relationship with recurrence. Similar results were obtained for DFS after PSM by Kaplan-Meier analysis (Figure 2) and by univariate and multivariate Cox regression analyses (Table 6). 
Table 2 Distribution of VATS segmentectomy and lobectomy procedures

\begin{tabular}{lcc}
\hline Variable & Segmentectomy $(\mathrm{n}=41)$ & Lobectomy $(\mathrm{n}=122)$ \\
\hline Left upper lobe & 22 & 33 \\
S1+S2 & 4 & \\
S1+S2+S3 & 15 & 17 \\
S4+S5 & 3 & \\
Left lower lobe & 4 & \\
S6 & 2 \\
S8 & 1 \\
S9 & 1 \\
Right upper lobe & 6 \\
S1+S2 & 2 \\
S1 & 1 \\
S2 & 2 \\
S3 & 1 \\
Right middle lobe & 0 \\
Right lower lobe & 9 \\
S6 & 1 \\
S6+S10 & 1 \\
S8 & 1 \\
S9 & 1 \\
\hline
\end{tabular}

\section{Discussion}

In 1939, Churchill and Belsey first reported 86 cases of lingulectomies for the treatment of bronchiectasis (9). During the following decades, thoracic surgeons performed segmentectomy to treat primary lung cancers. However, in 1995, the LCSG reported a prospective, randomized, multi-institutional trial comparing sublobar resection with lobectomy for T1N0 NSCLC and showed that limited resection was associated with higher death rate and recurrence than standard lobectomy (1). After that, segmentectomy was just performed for patients with limited cardiopulmonary reserve or significant comorbidities who were unable to tolerate lobectomy. However, there had been criticism of the LCSG study; there were potential biases in the LCSG study, because segmentectomy and wedge
Table 3 Postoperative complications by group

\begin{tabular}{|c|c|c|c|}
\hline Complications & $\begin{array}{l}\text { Segmentectomy } \\
\qquad(n=41)(\%)\end{array}$ & $\begin{array}{l}\text { Lobectomy } \\
(n=122)(\%)\end{array}$ & $\begin{array}{c}\mathrm{P} \\
\text { value }\end{array}$ \\
\hline Pneumonia & $1(2.4)$ & $3(2.5)$ & 1.000 \\
\hline Atrial fibrillation & $2(4.9)$ & $6(4.9)$ & 1.000 \\
\hline $\begin{array}{l}\text { Prolonged air leakage } \\
(\mathrm{d}>7)\end{array}$ & $4(9.8)$ & $6(4.9)$ & 0.272 \\
\hline Chylothorax & $0(0)$ & $2(1.6)$ & 1.000 \\
\hline Vocal cord paralysis & 0 & $1(0.8)$ & 1.000 \\
\hline Bronchopleural fistula & $0(0)$ & $0(0)$ & $\mathrm{N} / \mathrm{A}$ \\
\hline Wound infection & $0(0)$ & $1(0.8)$ & 1.000 \\
\hline $\begin{array}{l}\text { Acute cerebral } \\
\text { infarction }\end{array}$ & $0(0)$ & $1(0.8)$ & 1.000 \\
\hline Total & $6^{A}(14.6)$ & $19^{B}(15.6)$ & 1.000 \\
\hline
\end{tabular}

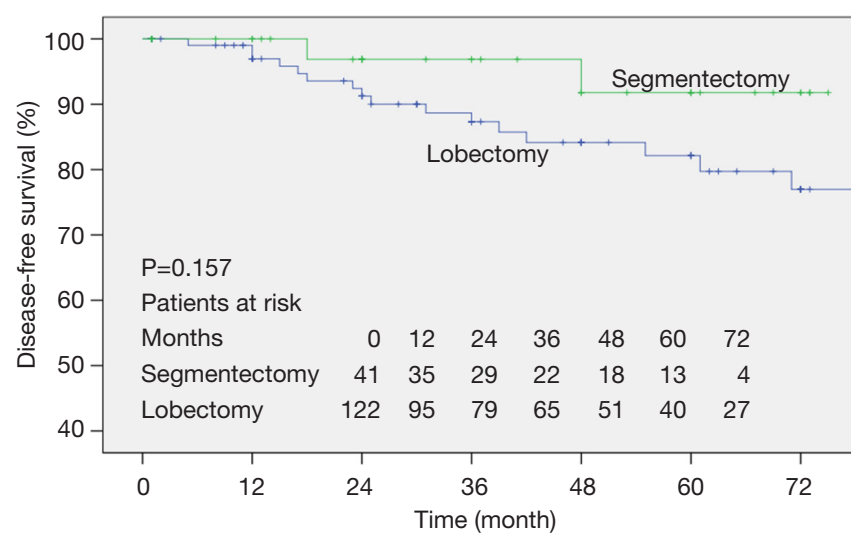

Figure 1 Association of type of operation with disease-free survival.

resection were not analyzed separately. Smith reported that wedge resection caused a higher recurrence rate than segmentectomy (2). In the last decades, lung cancers are increasingly being diagnosed in the early stage (10). Therefore, VATS segmentectomy has again become the focus of discussion for stage IA NSCLC. However, VATS segmentectomy has continued to be an area of controversy (3-6), because of the complexity of the procedure and the uncertainty about long-term outcomes.

The present study compared outcomes between patients 
Table 4 Univariate and multivariate Cox regression analyses for DFS of all patients

\begin{tabular}{|c|c|c|c|c|}
\hline Variables & \multicolumn{2}{|c|}{ Univariate } & \multicolumn{2}{|c|}{ Multivariate } \\
\hline \multicolumn{5}{|l|}{ Age, years } \\
\hline$<70$ & Reference & - & Reference & - \\
\hline$\geq 70$ & $0.510(0.202-1.867)$ & 0.390 & $0.527(0.160-1.733)$ & 0.292 \\
\hline Male & Reference & - & Reference & - \\
\hline Female & $0.432(0.162-1.153)$ & 0.094 & $0.771(0.174-3.491)$ & 0.732 \\
\hline \multicolumn{5}{|l|}{ T stage } \\
\hline Tis $+\mathrm{T} 1$ & Reference & - & Reference & - \\
\hline No & Reference & - & Reference & - \\
\hline $\mathrm{N} 1+\mathrm{N} 2$ & 7.845 (2.935-20.968) & $<0.001$ & $6.2 \mathrm{E}+5(0-2.2 \mathrm{E}+83)$ & 0.905 \\
\hline \multicolumn{5}{|l|}{ TNM stage } \\
\hline $0+1$ & Reference & - & Reference & - \\
\hline II+III & $5.894(2.208-15.736)$ & $<0.001$ & $0(0-4.4 \mathrm{E}+74)$ & 0.922 \\
\hline \multicolumn{5}{|l|}{ Smoking index } \\
\hline$<400$ & Reference & - & Reference & - \\
\hline$\geq 400$ & $2.003(0.776-5.172)$ & 0.151 & $1.798(0.406-7.972)$ & 0.440 \\
\hline Upper lobe & Reference & - & Reference & - \\
\hline Middle lobe & Reference & - & Reference & - \\
\hline Lower lobe & $0.935(0.560-1.560)$ & 0.796 & $0.936(0.550-1.593)$ & 0.807 \\
\hline \multicolumn{5}{|l|}{ Previous cancer } \\
\hline Yes & Reference & - & Reference & - \\
\hline No & $0.899(0.206-3.920)$ & 0.888 & $0.814(0.150-4.420)$ & 0.811 \\
\hline \multicolumn{5}{|l|}{ Type of operation } \\
\hline Segmentectomy & Reference & - & Reference & - \\
\hline Lobectomy & $0.362(0.083-1.576)$ & 0.176 & $0.410(0.051-3.282)$ & 0.401 \\
\hline
\end{tabular}


Table 5 Characteristics of the patients after propensity score matching

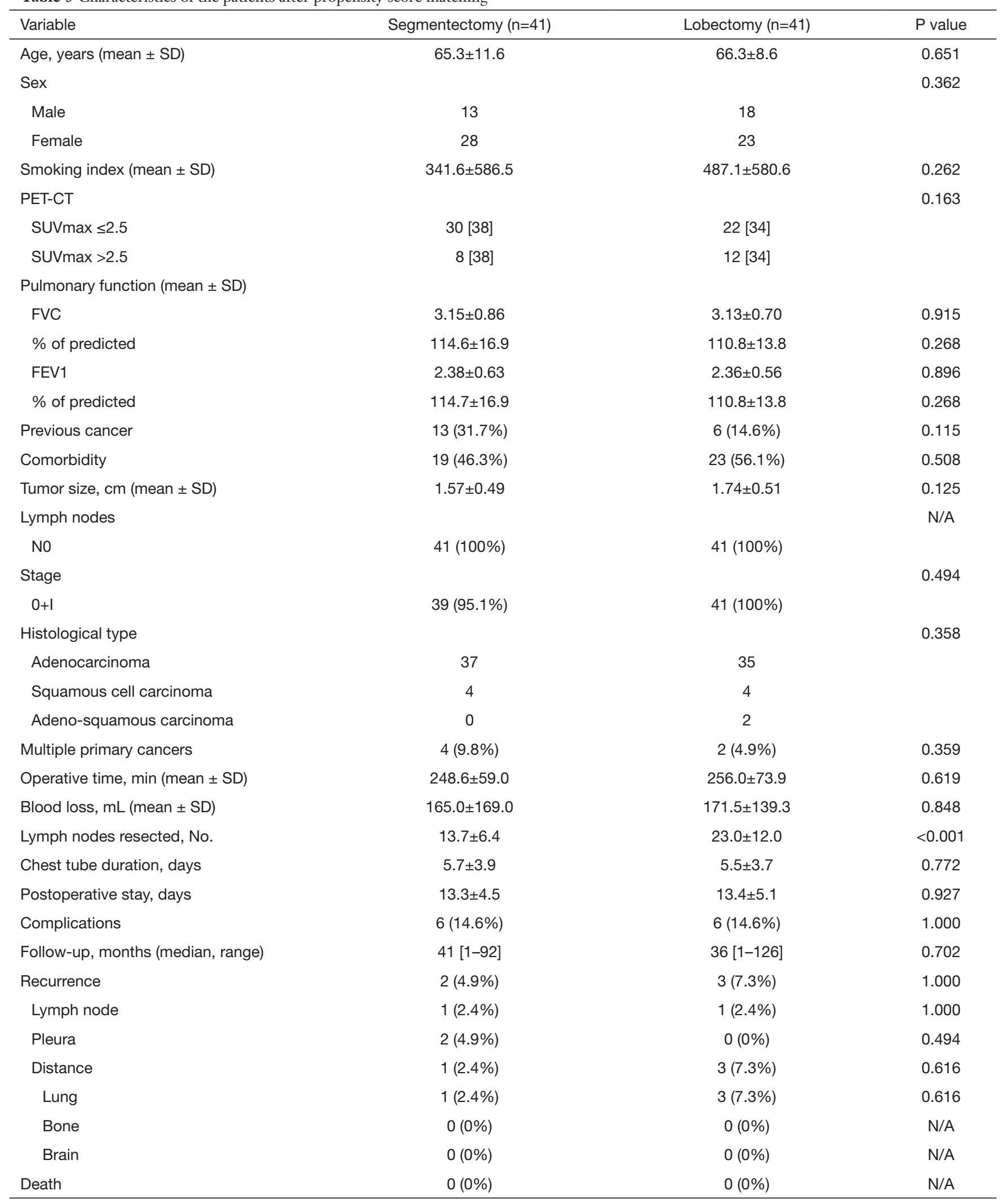




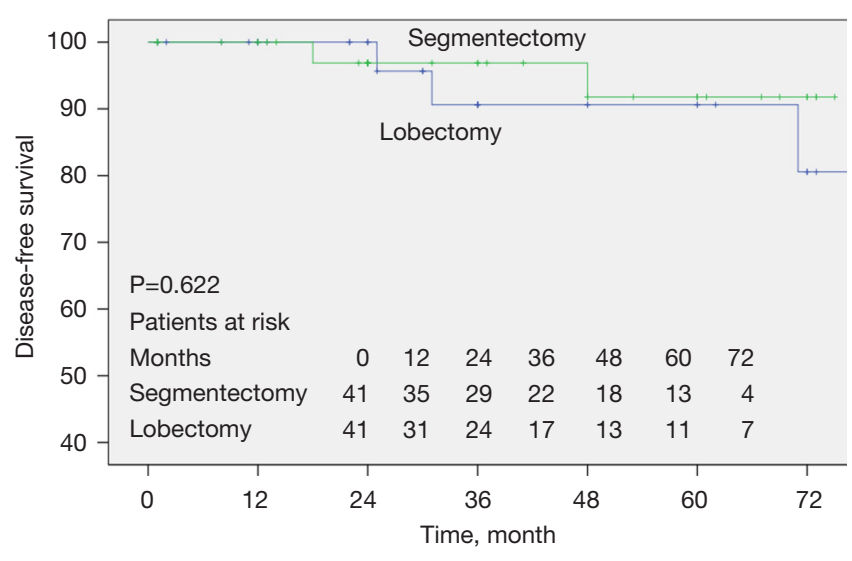

Figure 2 Association of type of operation with disease-free survival after propensity score matching.

who underwent lobectomy and segmentectomy for clinical stage IA NSCLC. In all cohorts, when the clinicopathological factors were not adjusted, the recurrence rate and death rate of the segmentectomy group were not significantly different from those of the lobectomy group. Univariate and multivariate Cox regression analyses showed that type of operation had no relationship to prognosis. However, there were some significant differences between the two groups with the same inclusion criteria previously described. Patients who underwent segmentectomy had a lower SUVmax value and higher previous cancer rate. During operation, wedge resection was selected to distinguish the histological type first, and then it was decided whether to perform radical excision. However, wedge resection is sometimes inappropriate technically. In order to minimize trauma, segmentectomy is acceptable instead of lobectomy. We performed segmentectomy for patients with smaller tumor size than patients undergoing lobectomy, because we were still skeptical about the reliability of segmentectomy. The reason why the patients who underwent lobectomy had pathologically more advanced stages might be the selection bias before the operation, including the SUVmax value and tumor size $(11,12)$. Since there were no significant differences in operative time, blood loss, chest tube drainage days, postoperative hospital stay, and postoperative complications between the segmentectomy and lobectomy groups, VATS segmentectomy can be considered an acceptable method based on the short-term surgical results. To minimize patient selection bias, PSM analyses were performed. Potentially confounding variables such as sex, PET-CT, tumor size, lymph node metastasis, pathological stage, and previous cancer were matched in lobectomy and segmentectomy. There were no significant differences in clinical features, pathological factors, and prognosis between the segmentectomy and lobectomy groups after PSM.

Most previous studies that showed favorable outcomes with segmentectomy selected this procedure for T1N0M0 NSCLC of $2 \mathrm{~cm}$ or less (13-15). However, Landreneau et al. compared 312 segmentectomies with 312 lobectomies including NSCLC of 2 to $3 \mathrm{~cm}$, and they suggested that segmentectomy appeared to offer comparable local control and the opportunity for prolonged disease-free and OSs that were not significantly different from those of lobectomy (16). Tsutani et al. suggested that the indication for segmentectomy for stage IA pulmonary adenocarcinoma depended on the solid tumor size and FDG-PET/CT findings (17), which means that whole tumor size of 2 to $3 \mathrm{~cm}$ is not a surgical contraindication for segmentectomy. In the present study, 87 NSCLCs of 2 to $3 \mathrm{~cm}$ were included, and 8 of them underwent segmentectomy, including 6 left upper lobe $\mathrm{S} 1+2+\mathrm{S} 3$ segmentectomies, 1 left lower lobe S6 segmentectomy, and 1 right upper lobe S3 segmentectomy; the other 79 cases underwent lobectomy. The width of the resection margin from the tumor was also an important factor affecting the recurrence rate, which should be more than $2 \mathrm{~cm}(18,19)$. In our strategy, when the resection margin of segmentectomy was less than $2 \mathrm{~cm}$ on intraoperative evaluation, multiple segmentectomies or conversion to lobectomy was considered. The recurrence rate of segmentectomy in the present study was $4.9 \%$, which was acceptable compared to previous reports $(3,16,17)$.

This study has some limitations. First, there was the potential selection bias, given the nature of a retrospective study. Although PSM was used to minimize selection bias between the two groups, it was impossible to eliminate all bias. Thoracic surgeons decided the type of operation according to comprehensive factors based on their experience, especially during the operation. That is the reason why it was almost impossible to control all factors that could cause bias. Second, the number of cases in the two groups was not great, which might also bias the results. Third, lung function was not routinely re-checked after the operation; thus, it was not possible to evaluate the differences in lung function between segmentectomy and lobectomy, as Charloux et al. reported (20).

In conclusion, perioperative and oncological outcomes of VATS segmentectomy are similar to those of VATS lobectomy for patients with clinical stage IA NSCLC. VATS segmentectomy can be one of the surgical procedures that is appropriate for patients with clinical stage IA NSCLC. 
Table 6 Univariate and multivariate Cox regression analyses after PSM

\begin{tabular}{|c|c|c|c|c|}
\hline Variables & \multicolumn{2}{|c|}{ Univariate } & \multicolumn{2}{|c|}{ Multivariate } \\
\hline \multicolumn{5}{|l|}{ Age, years } \\
\hline$<70$ & Reference & - & Reference & - \\
\hline$\geq 70$ & $1.243(0.207-7.480)$ & 0.812 & $0.272(0.010-7.318)$ & 0.438 \\
\hline Male & Reference & - & Reference & - \\
\hline Female & $0.146(0.016-1.318)$ & 0.087 & $0.095(0-92.24)$ & 0.502 \\
\hline \multicolumn{5}{|l|}{ T stage } \\
\hline Tis $+\mathrm{T} 1$ & Reference & - & Reference & - \\
\hline No & Reference & - & Reference & - \\
\hline $\mathrm{N} 1+\mathrm{N} 2$ & $\mathrm{~N} / \mathrm{A}$ & N/A & N/A & N/A \\
\hline \multicolumn{5}{|l|}{ TNM stage } \\
\hline $0+1$ & Reference & - & Reference & - \\
\hline II+III & $0.046(0-6.9 \mathrm{E}+6)$ & 0.749 & $0(0-N / A)$ & 0.989 \\
\hline \multicolumn{5}{|l|}{ Smoking index } \\
\hline$<400$ & Reference & - & Reference & - \\
\hline$\geq 400$ & $6.053(0.672-54.56)$ & 0.108 & 2.471 (0.003-2115) & 0.793 \\
\hline Upper lobe & Reference & - & Reference & - \\
\hline Middle lobe & Reference & - & Reference & - \\
\hline Lower lobe & $1.476(0.597-3.644)$ & 0.399 & $7.719(0.489-121.9)$ & 0.147 \\
\hline \multicolumn{5}{|l|}{ Previous cancer } \\
\hline Yes & Reference & - & Reference & - \\
\hline No & $0.986(0.107-9.10)$ & 0.990 & $1.674(0.027-105.4)$ & 0.807 \\
\hline \multicolumn{5}{|l|}{ Type of operation } \\
\hline Segmentectomy & Reference & - & Reference & - \\
\hline Lobectomy & $0.637(0.104-3.888)$ & 0.625 & $0.325(0.015-7.012)$ & 0.473 \\
\hline
\end{tabular}

PSM, propensity score matching. 


\section{Acknowledgements}

None.

\section{Footnote}

Conflicts of Interest: The authors have no conflicts of interest to declare.

Ethical Statement: This study was approved by The Committee of Medical Ethics of Hirosaki University Graduate School of Medicine, Hirosaki, Japan (approval number 2017-1155).

\section{References}

1. Ginsberg RJ, Rubinstein LV. Randomized trial of lobectomy versus limited resection for T1 N0 non-small cell lung cancer. Lung Cancer Study Group. Ann Thorac Surg 1995;60:615-22; discussion 622-3.

2. Smith CB, Swanson SJ, Mhango G, et al. Survival after segmentectomy and wedge resection in stage I non-smallcell lung cancer. J Thorac Oncol 2013;8:73-8.

3. Hwang Y, Kang CH, Kim HS, et al. Comparison of thoracoscopic segmentectomy and thoracoscopic lobectomy on the patients with non-small cell lung cancer: a propensity score matching study. Eur J Cardiothorac Surg 2015;48:273-8.

4. Bedetti B, Bertolaccini L, Rocco R, et al. Segmentectomy versus lobectomy for stage I non-small cell lung cancer: a systematic review and meta-analysis. J Thorac Dis 2017;9:1615-23.

5. Schuchert MJ, Pettiford BL, Keeley S, et al. Anatomic segmentectomy in the treatment of stage I non-small cell lung cancer. Ann Thorac Surg 2007;84:926-32; discussion 932-3.

6. Khullar OV, Liu Y, Gillespie T, et al. Survival After Sublobar Resection versus Lobectomy for Clinical Stage IA Lung Cancer: An Analysis from the National Cancer Data Base. J Thorac Oncol 2015;10:1625-33.

7. Whitson BA, Groth SS, Andrade RS, et al. Survival after lobectomy versus segmentectomy for stage I non-small cell lung cancer: a population-based analysis. Ann Thorac Surg 2011;92:1943-50.

8. Detterbeck FC, Boffa DJ, Kim AW, et al. The Eighth Edition Lung Cancer Stage Classification. Chest 2017;151:193-203.

9. Churchill ED, Belsey R. Segmental pneumonectomy in bronchiectasis: the lingula segment of the left upper lobe. Ann Surg 1939;109:481-99.

10. Goya T, Asamura H, Yoshimura H, et al. Prognosis of 6644 resected non-small cell lung cancers in Japan: a Japanese lung cancer registry study. Lung Cancer 2005;50:227-34.
11. Vansteenkiste J, Fischer BM, Dooms C, et al. Positronemission tomography in prognostic and therapeutic assessment of lung cancer: systematic review. Lancet Oncol 2004;5:531-40.

12. Hung JJ, Yeh YC, Jeng WJ, et al. Factors predicting occult lymph node metastasis in completely resected lung adenocarcinoma of $3 \mathrm{~cm}$ or smaller. Eur J Cardiothorac Surg 2016;50:329-36.

13. Okada M, Yoshikawa K, Hatta T, et al. Is segmentectomy with lymph node assessment an alternative to lobectomy for non-small cell lung cancer of $2 \mathrm{~cm}$ or smaller? Ann Thorac Surg 2001;71:956-60; discussion 961.

14. Yoshikawa K, Tsubota N, Kodama K, et al. Prospective study of extended segmentectomy for small lung tumors: the final report. Ann Thorac Surg 2002;73:1055-8; discussion 1058-9.

15. Okada M, Koike T, Higashiyama M, et al. Radical sublobar resection for small-sized non-small cell lung cancer: a multicenter study. J Thorac Cardiovasc Surg 2006;132:769-75.

16. Landreneau RJ, Normolle DP, Christie NA, et al. Recurrence and survival outcomes after anatomic segmentectomy versus lobectomy for clinical stage I non-small-cell lung cancer: a propensity-matched analysis. J Clin Oncol 2014;32:2449-55.

17. Tsutani Y, Miyata Y, Nakayama H, et al. Oncologic outcomes of segmentectomy compared with lobectomy for clinical stage IA lung adenocarcinoma: propensity score-matched analysis in a multicenter study. J Thorac Cardiovasc Surg 2013;146:358-64.

18. Sienel W, Stremmel C, Kirschbaum A, et al. Frequency of local recurrence following segmentectomy of stage IA nonsmall cell lung cancer is influenced by segment localisation and width of resection margins--implications for patient selection for segmentectomy. Eur J Cardiothorac Surg 2007;31:522-7; discussion 527-8.

19. Swanson SJ. Video-assisted thoracic surgery segmentectomy: the future of surgery for lung cancer? Ann Thorac Surg 2010;89:S2096-7.

20. Charloux A, Quoix E. Lung segmentectomy: does it offer a real functional benefit over lobectomy? Eur Respir Rev 2017;26.

Cite this article as: Song CY, Sakai T, Kimura D, Tsushima T, Fukuda I. Comparison of perioperative and oncological outcomes between video-assisted segmentectomy and lobectomy for patients with clinical stage IA non-small cell lung cancer: a propensity score matching study. J Thorac Dis 2018;10(8):4891-4901. doi: 10.21037/jtd.2018.07.133 